\title{
Systems Engineering approach for the concept design of the Crystal Eye detector
}

\author{
F. Renno ${ }^{1}$ F. Barbato ${ }^{2}$ - G. Barbarino ${ }^{2}$ D. Marzullo ${ }^{1}$ R. Guida ${ }^{1}$. S. Papa ${ }^{1}$
}

Received: 10 March 2020 / Accepted: 23 September 2020 / Published online: 12 October 2020

(c) The Author(s) 2020

\begin{abstract}
Main targets of this activity research are the making and the optimization of new detectors by means of the Systems Engineering methods. With the observation of the gravitational wave event of August 17th, 2017 and then with those of the extragalactic neutrino of September 22nd, the Multimessenger Astrophysics era began. It is a new way of exploring the Universe, powered by globally coordinated observations of several experiments. So, new $X$ and gamma rays' detectors solutions are needed in order to provide competitive results in the energy range $10 \mathrm{keV}-10 \mathrm{meV}$. Here is briefly described how the Systems Engineering can improve the development of the proposal of a new technique: The Crystal Eye, a wide field of view detector with a good spatial resolution obtained thanks to a high pixelation.
\end{abstract}

Keywords Systems Engineering · Optimization $\cdot$ Crystal Eye $\cdot$ Detectors $\cdot$ Astrophysics

\section{The Crystal Eye proposal}

Multimessenger Astrophysics is a new way of exploring the Universe, powered by globally coordinated observations of cosmic rays, neutrinos, gravitational waves, and electromagnetic radiation across a broad range of wavelengths. A long-standing astrophysical paradigm is that collisions, or mergers, of two neutron stars create highly relativistic and collimated jets that power $\gamma$-ray bursts of short duration. The observational support for this model, however, was only indi-

$凶$ F. Renno

fabrizio.renno@unina.it

F. Barbato

felicia.barbato@unina.it

G. Barbarino

giancarlo.barbarino@unina.it

D. Marzullo

domenico.marzullo@unina.it

R. Guida

riccardo.guida@euroavia.eu

S. Papa

stefpapa@unina.it

1 Fraunhofer JL Ideas-Department of Industrial Engineering, University of Naples Federico II, Naples, Italy

2 Department of Physics, University of Naples Federico II, Naples, Italy rect until August 2017, when the electromagnetic counterpart associated with the gravitational-wave event GW170817 was observed. The main currently active experiments in orbit able to observe the gamma and X-ray range are Chandra, XMMNewton and Fermi [5]. Despite their detection capability and their major observations, they belong to an old concept of sky observatories. Crystal Eye is a new detector proposal for the future exploration of the Universe. It will be something new, a crossover technology, exactly in the middle between the all sky monitors and the telescopes: a wide field of view observatory ( $2 \pi$ of local observation) in the energy range from $10 \mathrm{keV}$ to $10 \mathrm{meV}$ with a highly pixelated structure. In the past, it was impossible to use such an observation technique because of the size of the detectors and the consequent unaffordable costs. Today, this innovative observation technique is possible thanks to the use of new sensors and materials which allow the realization of a highly efficient, low cost, compact device. So, a Crystal Eye pathfinder made by four pixels has been designed and it will be launched in the early 2022 (January-April) aboard of the Space RIDER, an uncrewed reusable orbital spaceplane aiming to provide the European Space Agency (ESA) with affordable and routine access to space. The Crystal Eye prototype has been designed in order to be the lightest version able to provide basic results that will work as estimation of the physics potentiality of the full detector. The mission will follow a LEO orbit (like ISS orbit) for two months when it will come back at the base. An 


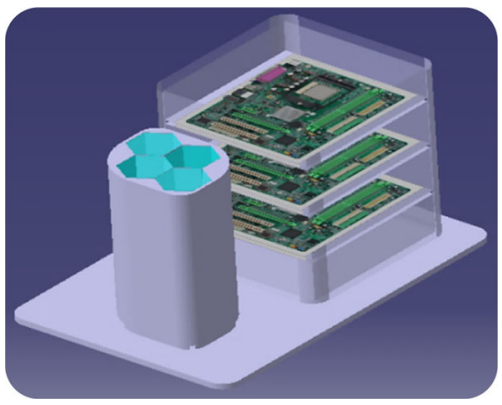

Fig. 1 First concept design of the Crystal Eye test case

SSD-disk will be installed to collect the data along the mission and at the same the time data will be sent to the ground base daily.

\section{Systems Engineering methods}

\subsection{Systems Engineering}

Systems Engineering (SE) is an interdisciplinary approach for the realization of successful systems. It focuses on defining the constraints and the functionality required in the early development cycle, documenting the needs and then proceeding with design synthesis and system validation. It is oriented to the improvement of the performance, test, manufacturing cost and schedule, training support and disposal. Systems Engineering integrates all the disciplines and speciality groups into a team effort forming a structured development process that proceeds from concept to production to operation. Systems Engineering considers both the business and the technical needs of all customers as its goal is to provide a quality product that meets the user needs [3].

\subsection{Systems Engineering for the Crystal Eye proposal}

According to SE principles, a first design concept of the fourpixel Crystal Eye test case has been developed (Fig. 1). The work has moved from the elicitation of high-level requirements and definition of the design parameters needed to achieve the required functionalities. The design parameters have been then associated to system components, which have been realized in CAD environment to generate the first design concept. The CAD model of the first Crystal Eye concept was built by a parametric approach and it was conceived to support structural/performance analyses, optimization processes and Verification and Validation (V\&V) $[1,2,4]$.

As the launch aboard of the Space RIDER implies high costs related to the weight of the components (thousands of euros for $1 \mathrm{~kg}$ ), it represents one of the main "variable" (parameters) to be considered. So, an optimization process

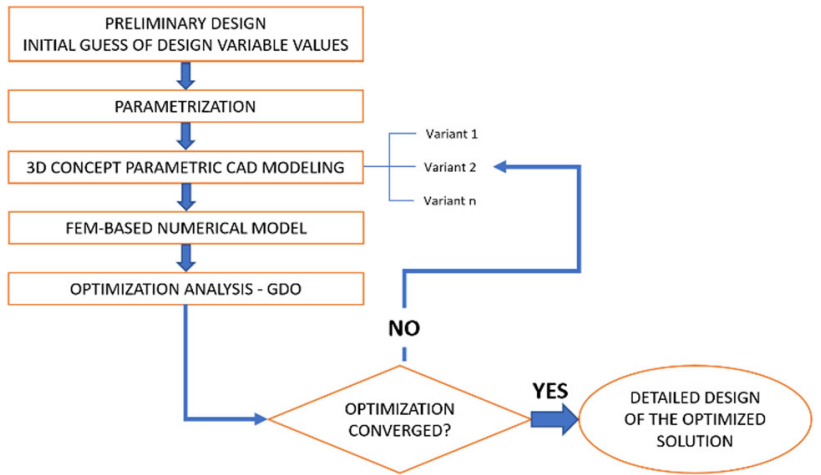

Fig. 2 Scheme of the optimization method conceived

has been conceived contemplating limited space, lightweight components and heavy loads during the launch operations.

\subsection{Optimization method}

An optimization process focused on the weight reduction and accurate dimensioning of all the components has been considered due to the high cost of the project. It is summarized in Fig. 2. Starting from the preliminary design, the main parameters have been identified and implemented in the CAD/FEM model, where the upper and lower limits for each variable have been defined, as well as the goal of the optimization (e.g.minimize weight, minimize Von Mises stress, reduce the deformation within certain limits, etc.). The optimization analysis is then performed by means of the "DesignXplorer" module available in ANSYS Workbench, which implements different optimization techniques related to the input/output variables. In the case of the Space RIDER, the Direct Optimization using the Adaptive Multiple Objective optimization method has been selected. The process ends when the optimization converges, and the resulting parameters are correctly verified and validated according to the requirements and the constraints.

\subsection{Scheme of the optimized model}

The process described in Fig. 2 has allowed to get a new scheme of the system that completely fits the requirements imposed for the Space RIDER launch. So, a simplified model of the first concept was designed in ANSYS, where a proper parametrization has been set, mainly regarding the position and the diameter of the fixation screws. Figure 3 shows the concept conceived after a first topological optimization, whereas the Fig. 4 shows the model designed in ANSYS, with the boundary conditions applied to the bolts locations and the loads used for the optimization studies (acceleration $=6 \mathrm{~g}$, in all directions). 


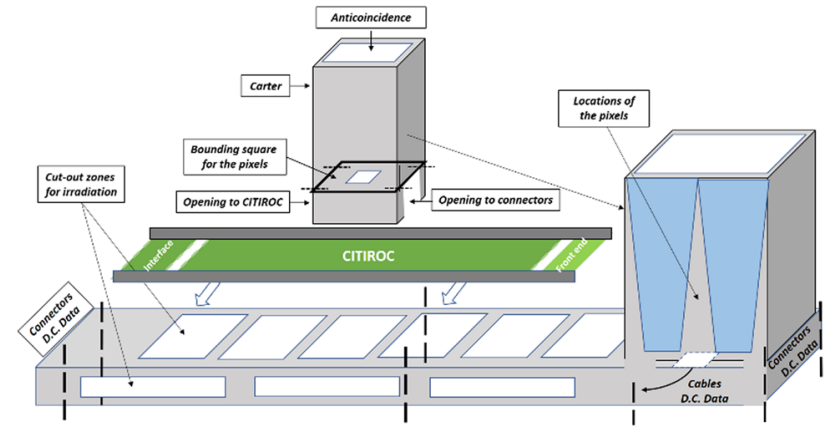

Fig. 3 Scheme of the model optimized with the design process
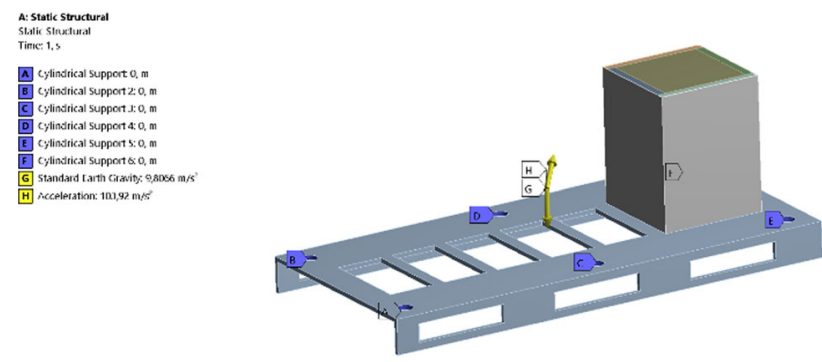

Fig. 4 Constraints and loads applied in ANSYS Mechanical

The screws diameters and their layout, i.e. the distance among the holes and their distances from the edges, have been considered as parameters in the Direct Optimization tool.

Figure 5 shows a screenshot of the ANSYS Workbench, where the Direct Optimization tool has been used to identify the possible configurations of the selected parameters with the objective to minimize the axial reaction forces to the screws.

Totally, 156 design configurations have been created and analysed in the Direct Optimization tool, among which, three candidate design points have been selected as possible optimized configurations (see Table 1).

Given the reaction forces results and the geometrical boundaries, the candidate point 3 has been selected (Fig. 6).

\section{Future works}

The virtual and the physical mock-ups of the four-pixel Crystal Eye test case will be realized in a short time. Afterwards, the needed simulation and testing phases (i.e. FEM, Multiphysical and Vibration analyses) will be carried on according to the scheduled time of the Space RIDER project.

Funding Open access funding provided by Università degli Studi di Napoli Federico II within the CRUI-CARE Agreement.

Open Access This article is licensed under a Creative Commons Attribution 4.0 International License, which permits use, sharing, adap-

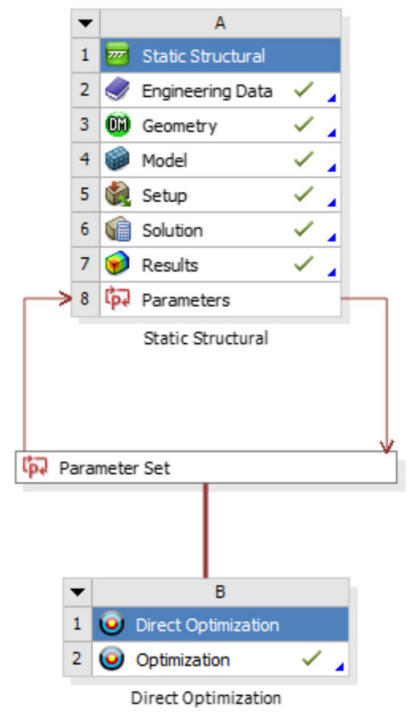

Fig. 5 Screenshot of ANSYS Workbench with parametrization and direct optimization tool

Table 1 Force reactions at bolt axis for three candidate points

\begin{tabular}{lllllll}
\hline \multicolumn{7}{c}{ Force reaction bolt axis (N) } \\
\cline { 2 - 7 } & Bolt 1 & Bolt 2 & Bolt 3 & Bolt 4 & Bolt 5 & Bolt 6 \\
\hline $\begin{array}{l}\text { Candidate point } \\
\quad\end{array}$ & -0.03 & 0.46 & 32.29 & 25.66 & 43.62 & -7 \\
$\begin{array}{l}\text { Candidate point } \\
2\end{array}$ & -0.04 & 0.45 & 30.75 & 26.92 & 46.2 & -9.29 \\
$\begin{array}{l}\text { Candidate point } \\
3\end{array}$ & -0.04 & 0.53 & 33.82 & 23.9 & 42.26 & -5.51 \\
\hline
\end{tabular}

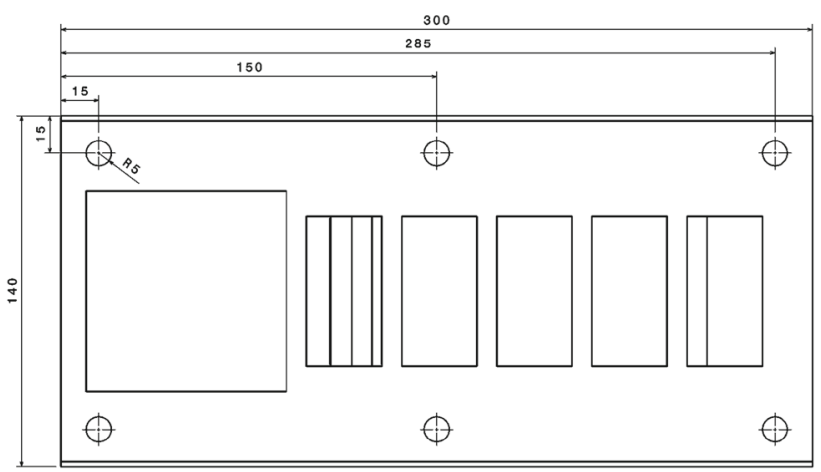

Fig. 6 Optimized attachment configuration

tation, distribution and reproduction in any medium or format, as long as you give appropriate credit to the original author(s) and the source, provide a link to the Creative Commons licence, and indicate if changes were made. The images or other third party material in this article are included in the article's Creative Commons licence, unless indicated otherwise in a credit line to the material. If material is not included in the article's Creative Commons licence and your intended use is not permitted by statutory regulation or exceeds the permitted use, you will need to obtain permission directly from the copy- 
right holder. To view a copy of this licence, visit http://creativecomm ons.org/licenses/by/4.0/.

\section{References}

1. Di Gironimo, G., et al.: Concept design of the DEMO divertor cassette-to-vacuum vessel locking system adopting a systems engineering approach. Fus. Eng. Des. 94, 1 (2015)

2. Di Gironimo, G., et al.: Iterative and participative axiomatic design process in complex mechanical assemblies: case study on fusion engineering. IJIDeM 9(4), 325-338 (2015)
3. Liu, D.: Systems Engineering Design Principles and Models. CRC Press Taylor \& Francis Group, Boca Raton (2016)

4. Mozzillo, R., et al.: Development of a master model concept for DEMO vacuum vessel. Fus. Eng Des. 112, 497-504 (2016)

5. Troja, E., et al.: The X-ray counterpart to the gravitational-wave event gw170817. Nature 551, 71 (2017)

Publisher's Note Springer Nature remains neutral with regard to jurisdictional claims in published maps and institutional affiliations. 